\title{
The non-invasive assessment of myocardial work by pressure-strain analysis: clinical applications
}

\author{
Dawud Abawi ${ }^{1} \cdot$ Tommaso Rinaldi $^{2} \cdot$ Alessandro Faragli $^{1,3,4,5} \cdot$ Burkert Pieske $^{1,3,4,5} \cdot$ Daniel A. Morris ${ }^{1}$. \\ Sebastian Kelle ${ }^{1,3,4} \cdot$ Carsten Tschöpe $^{1,3,6} \cdot$ Concetta Zito $^{2} \cdot$ Alessio Alogna ${ }^{1,3,5}$
}

Accepted: 28 April 2021 / Published online: 26 May 2021

(c) The Author(s) 2021

\begin{abstract}
Pressure-volume (PV) analysis is the most comprehensive way to describe cardiac function, giving insights into cardiac mechanics and energetics. However, PV analysis still remains a highly invasive and time-consuming method, preventing it from integration into clinical practice. Most of the echocardiographic parameters currently used in the clinical routine to characterize left ventricular (LV) systolic function, such as LV ejection fraction and LV global longitudinal strain, do not take the pressure developed within the LV into account and therefore fall too short in describing LV function as a hydraulic pump. Recently, LV pressure-strain analysis has been introduced as a new technique to assess myocardial work in a non-invasive fashion. This new method showed new insights in comparison to invasive measurements and was validated in different cardiac pathologies, e.g., for the detection of coronary artery disease, cardiac resynchronization therapy (CRT)-response prediction, and different forms of heart failure. Non-invasively assessed myocardial work may play a major role in guiding therapies and estimating prognosis. However, its incremental prognostic validity in comparison to common echocardiographic parameters remains unclear. This review aims to provide an overview of pressure-strain analysis, including its current application in the clinical arena, as well as potential fields of exploitation.
\end{abstract}

Keywords Non-invasive pressure-strain analysis $\cdot$ Myocardial work $\cdot$ Myocardial mechanics $\cdot$ Echocardiography $\cdot$ Strain . Speckle tracking

Alessio Alogna

alessio.alogna@charite.de

1 Department of Internal Medicine and Cardiology, Charité - Universitätsmedizin Berlin, Campus Virchow-Klinikum, Augustenburgerplatz 1, 13353 Berlin, Germany

2 Department of Clinical and Experimental Medicine, Cardiology Unit - University of Messina, Messina 98100, Italy

3 DZHK (German Centre for Cardiovascular Research), partner site Berlin, Germany

4 Department of Internal Medicine/Cardiology, Deutsches Herzzentrum Berlin, Augustenburgerplatz 1, 13353 Berlin, Germany

5 Berlin Institute of Health, Berlin, Germany

6 Berlin Institute of Health Center for Regenerative Therapies (BCRT), Charité - University Medicine Berlin, Campus Virchow Clinic, Augustenburgerplatz 1, 13353 Berlin, Germany

\section{Introduction}

The evaluation of left ventricular (LV) systolic function is of significant importance in all echocardiographic examinations. The most common parameter to assess systolic function is left ventricular ejection fraction (LVEF), whereas reduced LVEF is correlated with poor outcome [1-3]. However, LVEF is sensitive neither for the assessment of regional differences in myocardial function nor for diastolic dysfunction [4]. Strain analysis by speckle-tracking echocardiography (STE) has become more and more relevant in the evaluation of regional and global left ventricular function over the last years, primarily due to its ability to detect subclinical dysfunction in a variety of cardiac pathologies, including a condition of preserved LVEF [5-11]. A major limitation of myocardial strain-analysis lies in its load dependency, which can lead to misinterpretation of the actual myocardial contractility $[12,13]$.

Pressure-volume analysis, the gold standard in assessing ventricular function, delivers reasonably load-independent 
measures, as the end-systolic and end-diastolic pressurevolume relationships [14-17]. Furthermore, PV analysis gives insight into the ventricular-arterial coupling and offers information about myocardial energetics and efficiency. In terms of myocardial energetics, the total myocardial oxygen consumption per beat (MVO2) is described by the pressurevolume area (PVA), which is defined as the sum of stroke work (SW) and the potential energy (PE) [18-20]. Stroke work stands for the external work of the heart, meaning the energy which is required to eject blood into the vasculature system and is represented by the area of the pressurevolume loop [18]. PE is the potential myocardial work which is not liberated due to aortic valve closure and stored in the myofilaments (Fig. 1) [18]. Pressure-volume analysis has been proven to be an essential tool for the understanding of cardiovascular physiology and pathophysiology. However, due to its invasive nature and complexity, it has never been implemented into daily clinical practice.

Due to a further development in echocardiographic techniques including the implementation of volume and strain analysis, it became possible to estimate diastolic and systolic pressure volume relationships non-invasively [21, 22]. Recently, Russel et al. established a method to noninvasively assess myocardial work by obtaining pressurestrain-loops via STE based on a non-invasive approach to estimate left ventricular pressure (LVP) [22]. This review aims to provide an overview of the current state of research regarding this promising and holistic approach to non-invasively assess LV function.

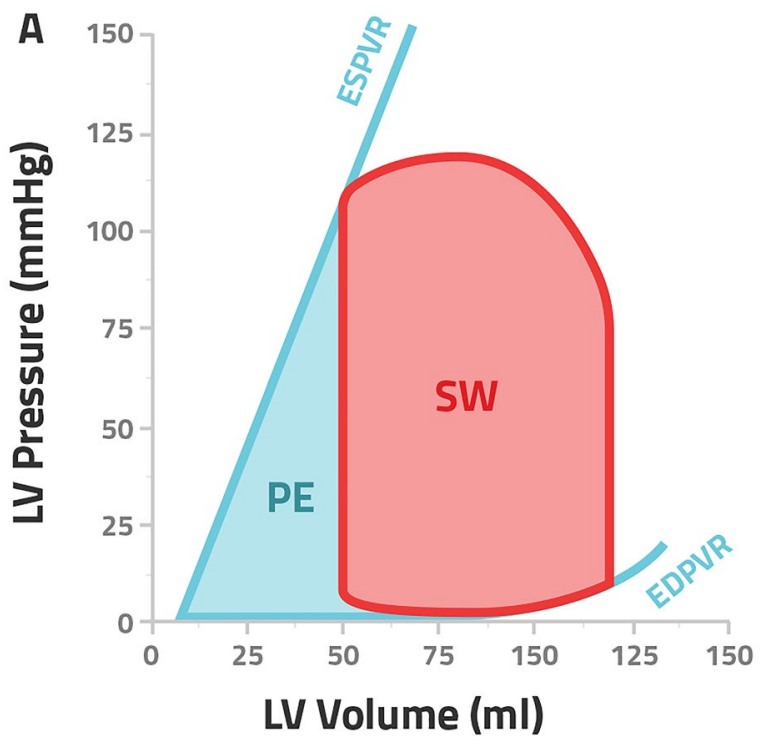

Fig. 1 Pressure-volume analysis; the sum of the potential energy (PE) and stroke work (SW) describes the pressure volume area (PVA), ESPVR end-systolic pressure volume relationship, EDPVR end-diastolic pressure-volume relationship

\section{Theoretical background and first clinical validation}

Whereas the pressure-volume loop describes the global myocardial work und function, also regional myocardial work has been studied in the form of pressure-length loops, obtained via micromanometers for the assessment of pressure, and implanted sonomicrometric crystals for the calculation of dimensions in animal models [23, 24].

The sonomicrometry technique, first introduced in 1956 by Rushmer et al. [25], has traditionally been used for the assessment of the dimensions of the heart [26, 27]. One of the first studies on the topic by Leraand et al. showed that ultrasonic elements sutured into the left myocardial wall of anesthetized dogs correctly measured local myocardial distances [28].

In analogy to the pressure-volume loop ability to reflect global myocardial oxygen consumption, Delhaas et al. could show that the regionally assessed stress-fiber strain area was not only able to reflect regional myocardial work but also regional oxygen demand [29].

Assessment of myocardial wall stress is difficult and not feasible in clinical practice. Studies by Skulstad and Urheim et al. could validate the invasively measured LV pressure as a surrogate of left ventricular wall stress [30, 31]. Furthermore, they demonstrated that regional myocardial work can be estimated from a combined measurement of invasively assessed LV pressure and myocardial strain by strain Doppler echocardiography (SDE) [30, 31]. Over a wide range of conditions, the SDE method correlated well with those measured by sonomicrometry [30,31], leading to a more non-invasive approach of determining myocardial work.

The new approach by Russel et al. goes even a step further by assessing myocardial work completely noninvasively. They combined segmental strain curves by speckle tracking echocardiography with an estimated LV pressure curve where the systolic cuff pressure is used as a surrogate of LV peak pressure [22]. The method was validated in a variety of pathologies showing a good correlation to invasive measurements [5, 13, 22, 32-38]. Similar to the pressure-volume loop, non-invasive pressure-strain loops were shown to reflect regional myocardial oxygen consumption and metabolism validated by ${ }^{18} \mathrm{~F}$-fluorodeoxyglucose positron emission tomography [22] (Table 1).

\section{Segmental work calculation}

In general, a vendor-specific module (EchoPAC Version $202, \mathrm{GE})$ is necessary to assess non-invasive myocardial work by combining left ventricular strain data obtained via speckle-tracking echocardiography with a non-invasively estimated LV pressure curve. 


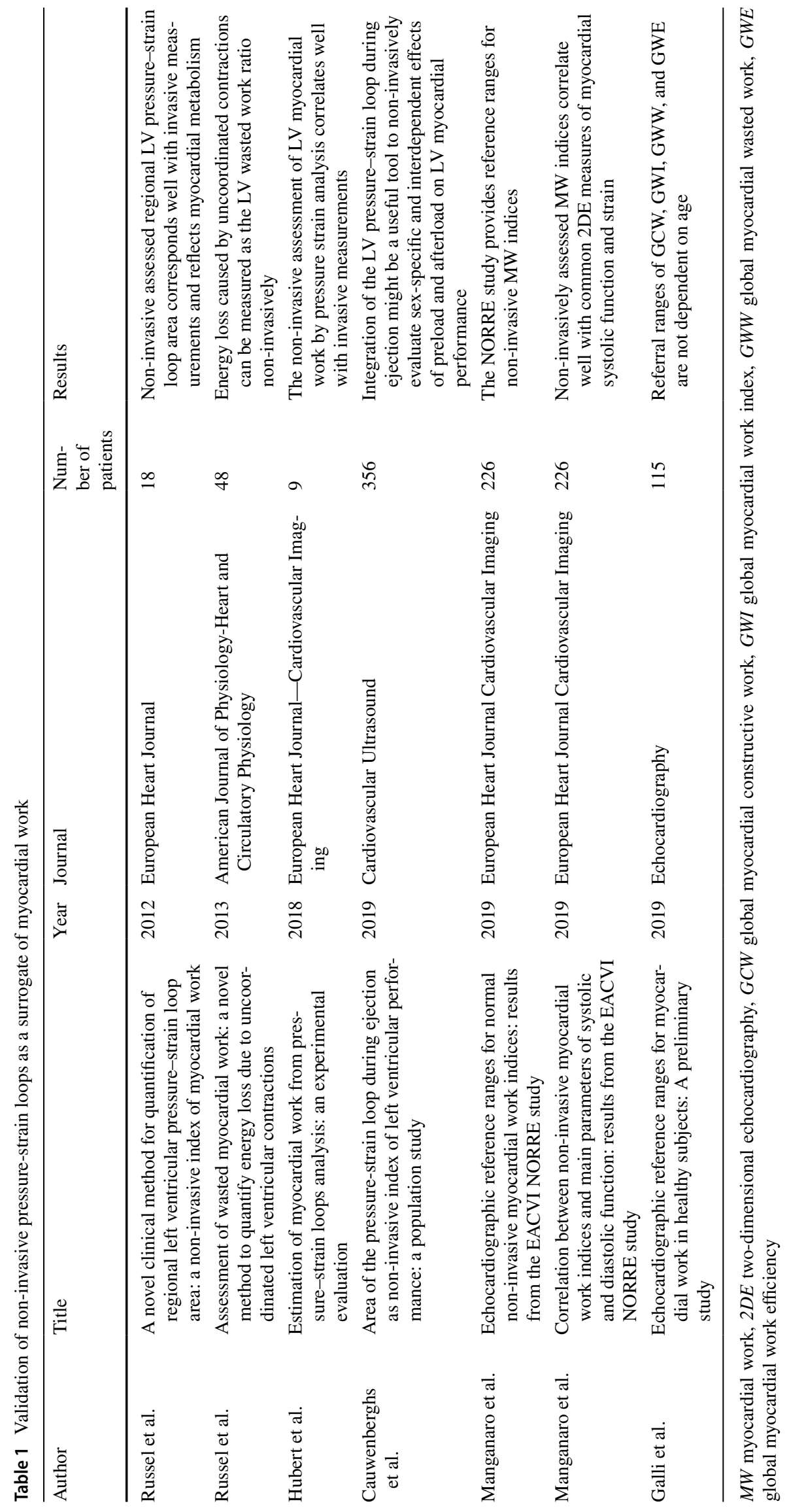


Practically, LV global longitudinal strain (GLS) has to be estimated by processing two-dimensional grayscale images acquired in the standard apical two-, three-, and four-chamber views at similar heart rate, depth, and a frame rate between 38 and 80 frames/s with an off-line dedicated software (Automated Functional Imaging; EchoPAC®, Version 202, GE).

The novelty of integrating non-invasive LV pressure is based on the creation of a normalized left ventricular pressure curve which was obtained by assembling invasive LVP measurements from patients in different conditions of inotropy [22]. All invasive LVP measurements were normalized to equal durations of isovolumetric contraction, ejection phase, and isovolumetric relaxation as well as the amplitudes of left ventricular peak pressure [22].

To then establish each patient's specific LVP curve, the normalized LVP curve has to be adjusted according to the patient's valvular event times assessed via echocardiography and the measured systolic cuff pressure, as a surrogate for LV peak pressure [22].

To determine valvular events, event timing can be derived by ECG-triggering or Doppler echocardiography. Electrical and mechanical phases should be aligned and can be manually adjusted. It is recommended to assess the blood pressure at the time of the examination (Fig. 2).

A 17-segment model is considered for the calculation of segmental myocardial work. However, segmental myocardial work is not assessed by calculating the area of each segmental pressure strain loop, rather it is calculated as a function of time for the duration of the whole cardiac cycle [32]. In particular, according to Russel et al. the strain recordings have to be differentiated to acquire the corresponding strain rate, which is then multiplied with the instantaneous LV pressure, resulting in a measure of power [32]. To finally obtain myocardial work as a function of time, instantaneous power also has to be integrated over time (Fig. 3).

Global values of myocardial work are derived from the average of all segmental myocardial work values. Further parameters describe the relationship between positive and negative myocardial work according to their occurrence during the cardiac cycle (Fig. 4):

- Constructive myocardial work (GCW): positive segmental work performed during myocardial shortening in systole and negative segmental work during lengthening in isovolumic relaxation
Fig. 2 Practical assessment of myocardial work. a Global longitudinal strain is calculated through two-dimensional grayscale images acquired in the standard apical two-, three- and four-chamber views. b Visualization of the calculated strain measurements. c Determination of valvular event timings using pulsed-wave Doppler or ECG-triggering and insertion of systolic and diastolic blood pressure levels for myocardial work calculation. d Visualization of myocardial work via the pressure-strain loop a)

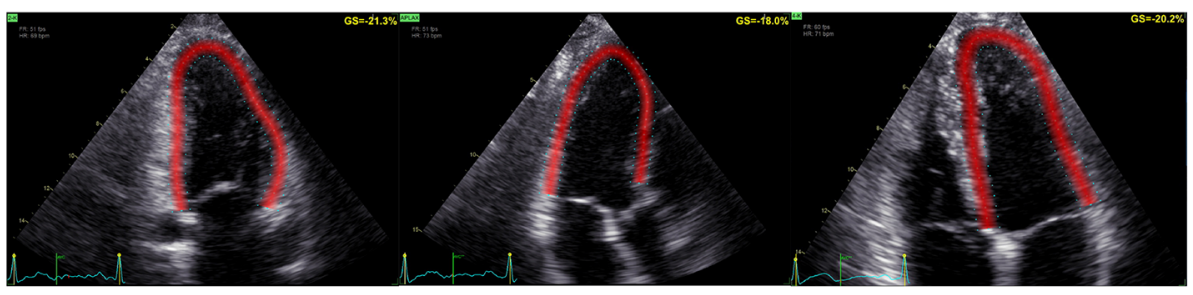

b)

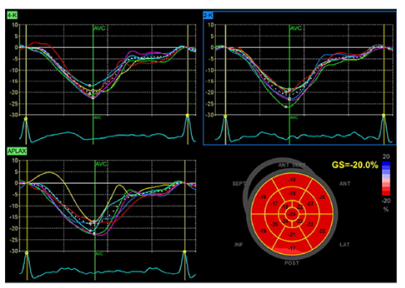

c)
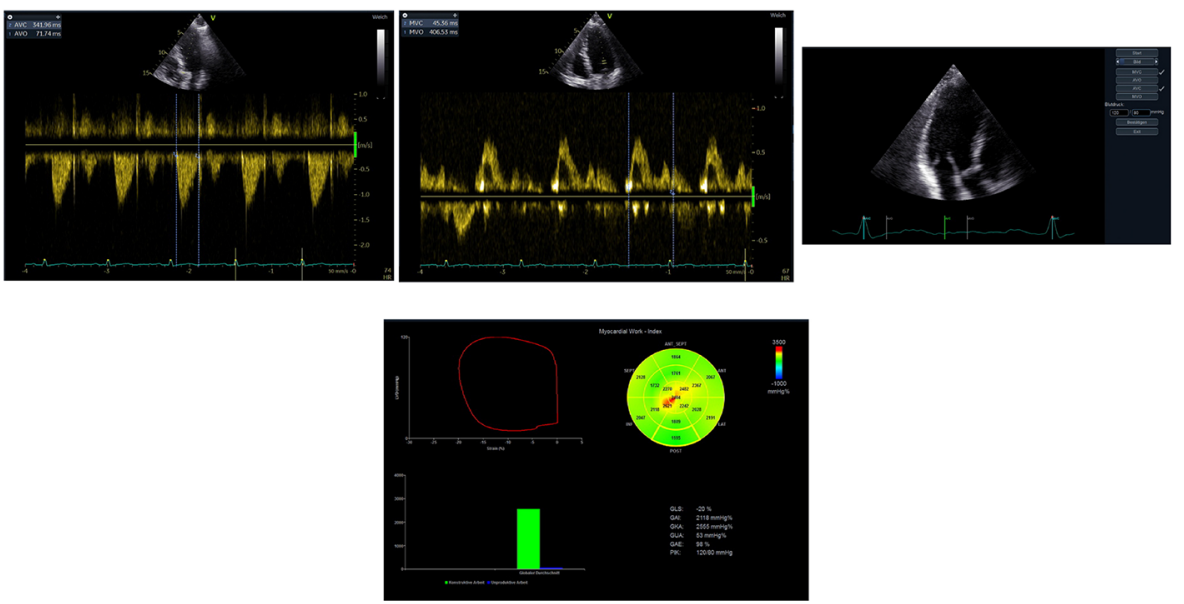

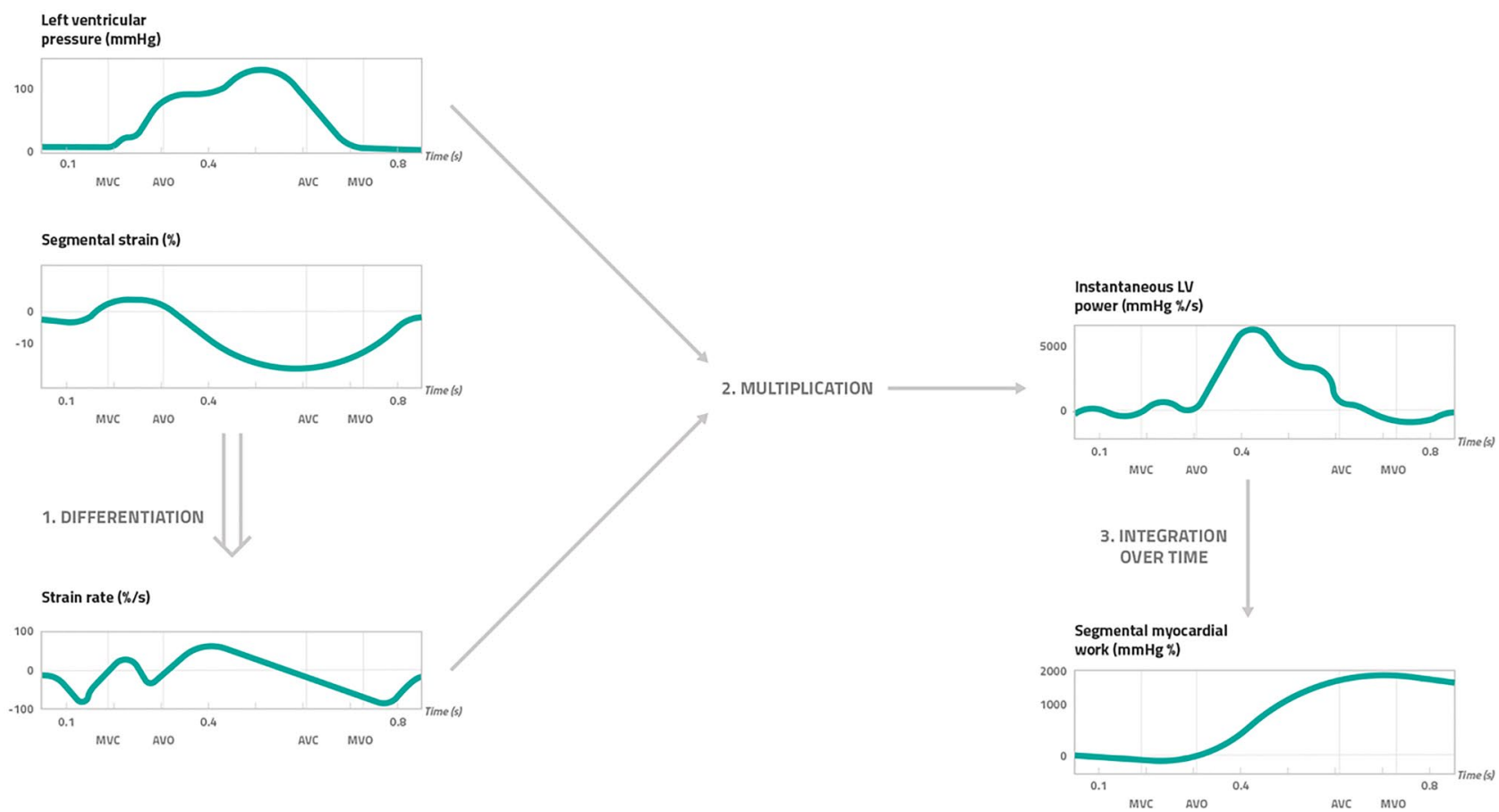

Fig. 3 Calculation of segmental myocardial work by pressure strainanalysis. Myocardial work by pressure strain analysis is calculated as a function of time throughout the cardiac cycle. The calculational steps include (1) the differentiation of the segmental strain (\%) in order to obtain the strain rate $(\% / \mathrm{s})$ which was then (2) multiplied with the left ventricular pressure $(\mathrm{mmHg})$. This results in a measure

- Wasted myocardial work (GWW): negative segmental work performed during lengthening in systole and positive segmental work performed during myocardial shortening in isovolumic relaxation

- Myocardial work efficiency (GWE): relationship between constructive work and the sum of constructive and wasted work

\section{Methods}

For this literature review, we searched MEDLINE (PubMed) and Embase, while clinicaltrials.gov was searched for ongoing clinical studies. The following search terms included in the title were used: "cardiac work," "constructive work," "global longitudinal strain," "global work index," "myocardial work," "myocardial work efficiency," "non-invasive myocardial work," in combination with "pressure-strain loops" in the title or abstract. Relevance and credibility of all the sources were considered, and the final decision on inclusion was reached through a consensus of the following screening authors: DA, TR, AF, and AA. Review articles, case reports, comments, and author replies were excluded. The cited references were published within the last 8 years. of instantaneous $\mathrm{LV}$ power ( $\mathrm{mmHg} \% / \mathrm{s})$. To finally obtain segmental myocardial work over time $(\mathrm{mmHg} \%)$, (3) the instantaneous LV power has to be integrated over time. MVC mitral valve closure, AVO atrial valve opening, AVC atrial valve closure, MVO mitral valve opening

A narrative synthesis with a tabulation system was used to analyze studies for their diverse research designs, methods, and implications.

\section{Clinical applications}

In the present review, we focused on the state of the art regarding non-invasive myocardial work assessment in several clinical fields: in CRT recipients, in patients with ischemic cardiac disease, mitral valve repair, heart failure with reduced (HFrEF) and those with heart failure and preserved ejection fraction (HFpEF) or HFpEF-like syndromes (hypertrophic cardiomyopathy, cardiac amyloidosis) (Fig. 5).

Furthermore, we searched for ongoing clinical trials focusing on non-invasive myocardial work assessed by pressure-strain loops.

\section{Cardiac resynchronization therapy}

Cardiac resynchronization therapy (CRT) is an effective therapy that aims to restore mechanical efficiency to the failing LV by resynchronizing the contraction of the left and right ventricle, resulting in a reduction of both morbidity and mortality [39]. According to the 2016 ESC guidelines for the 


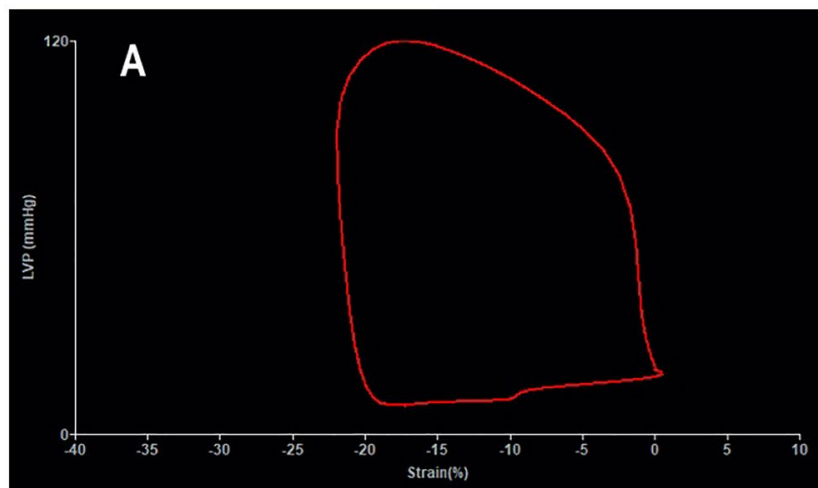

C

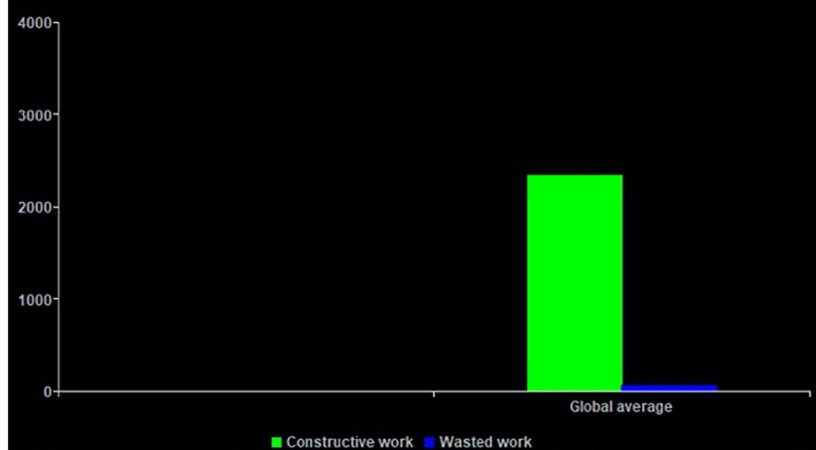

Fig. 4 Measurement of myocardial work indices by 2D echocardiography. A Left ventricular pressure-strain loop. B Bull's eye of global work index (GWI). C Bar graphs depicting global constructive work

diagnosis and treatment of heart failure, CRT is taken into account with a class I a recommendation in symptomatic patients with heart failure (HF) in sinus rhythm with a QRS duration $\geq 150 \mathrm{~ms}$ and left bundle branch block (LBBB) as well as with an LVEF $\leq 35 \%$ despite optimal medical therapy (OMT); in patients with HFrEF regardless of their NYHA class who indicate for ventricular pacing and high degree AV block (including patients with atrial fibrillation) [40].

However, the number of patients who do not respond to CRT remains high (30-35\%) [41]. Estimating CRT response is difficult as there is a lack of a globally accepted CRT response definition. Mostly, CRT response is identified by $a \geq 10-15 \%$ reduction of $L V$ end-systolic volume (LVESV), assessed by echocardiography 6 months after device implantation, as a sign of LV reverse remodeling [42]. Current patient selection criteria utilize the surface 12-lead ECG to identify electromechanical delay; here, the presence of left bundle branch block morphology is considered to be a predictor of response to CRT [39]. However, it is becoming increasingly clear that QRS duration is an inadequate predictor of CRT response, since LV dyssynchrony is not uncommon in patients with a narrow QRS, proving how electrical dyssynchrony does not always correlate to mechanical delay [43]. In such cases, mechanical dyssynchrony may be,

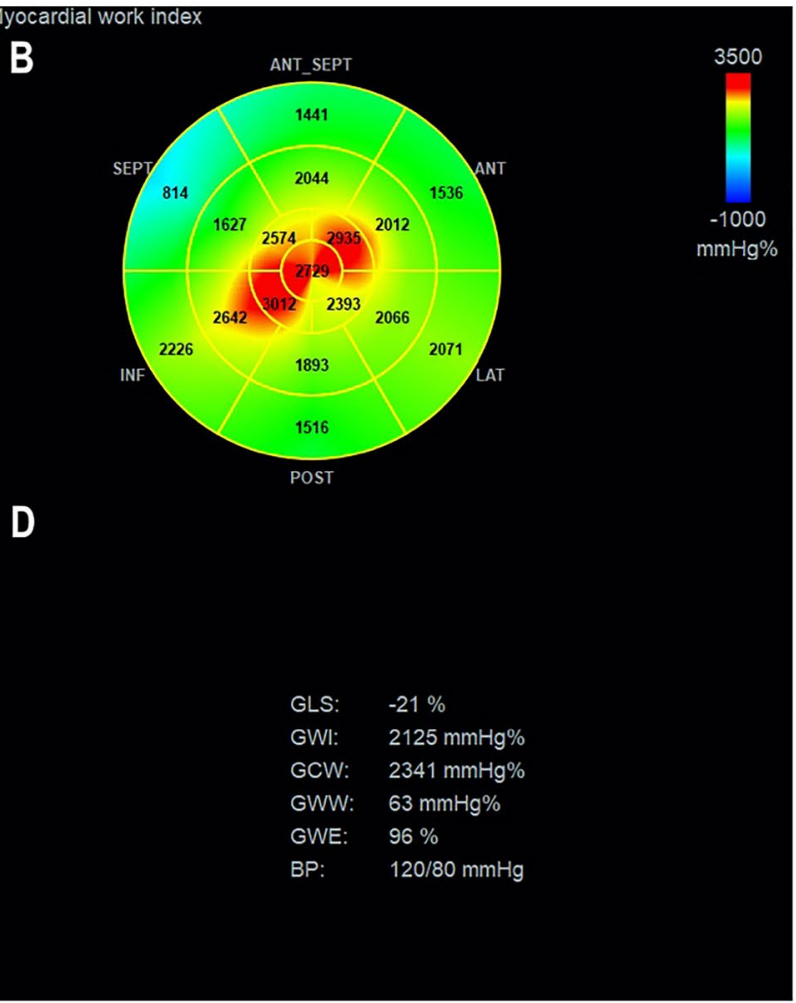

(GCW) and global wasted work (GWW). D Results from myocardial work analysis

in fact, the result of abnormalities in regional contractility of the LV and loading conditions which can be better assessed through further imaging techniques, rather than ECG. However, although some echocardiographic dyssynchrony parameters have proven to be valuable in predicting CRT response in numerous, single-center studies [44, 45], the multi-center PROSPECT trial showed how such predictors are burdened with low sensitivity and specificity [42]. Therefore, as of today, no imaging technique is accepted for the identification of CRT responders, as also pointed out by $2016 \mathrm{ESC}$ heart failure guidelines, which discourage the use of echocardiographic dyssynchrony criteria for the selection of patients [40]. Lastly, some clinical evaluations have also been proposed to detect CRT responders: male sex and ischemic etiology were shown as good predictors to less favorable CRT response [46, 47]. Nonetheless, when they were considered together with baseline LV volumes, they proved to be no longer independent variables [48]. Regarding the use of pressure-strain analysis, Galli et al. could show that GCW and GWW were associated with a positive response to CRT $[36,38]$. GCW and GWW at baseline were significantly higher in CRT-responders in comparison to non-responders. After 6 months of follow up, CRT responders presented an increase in GCW and a 


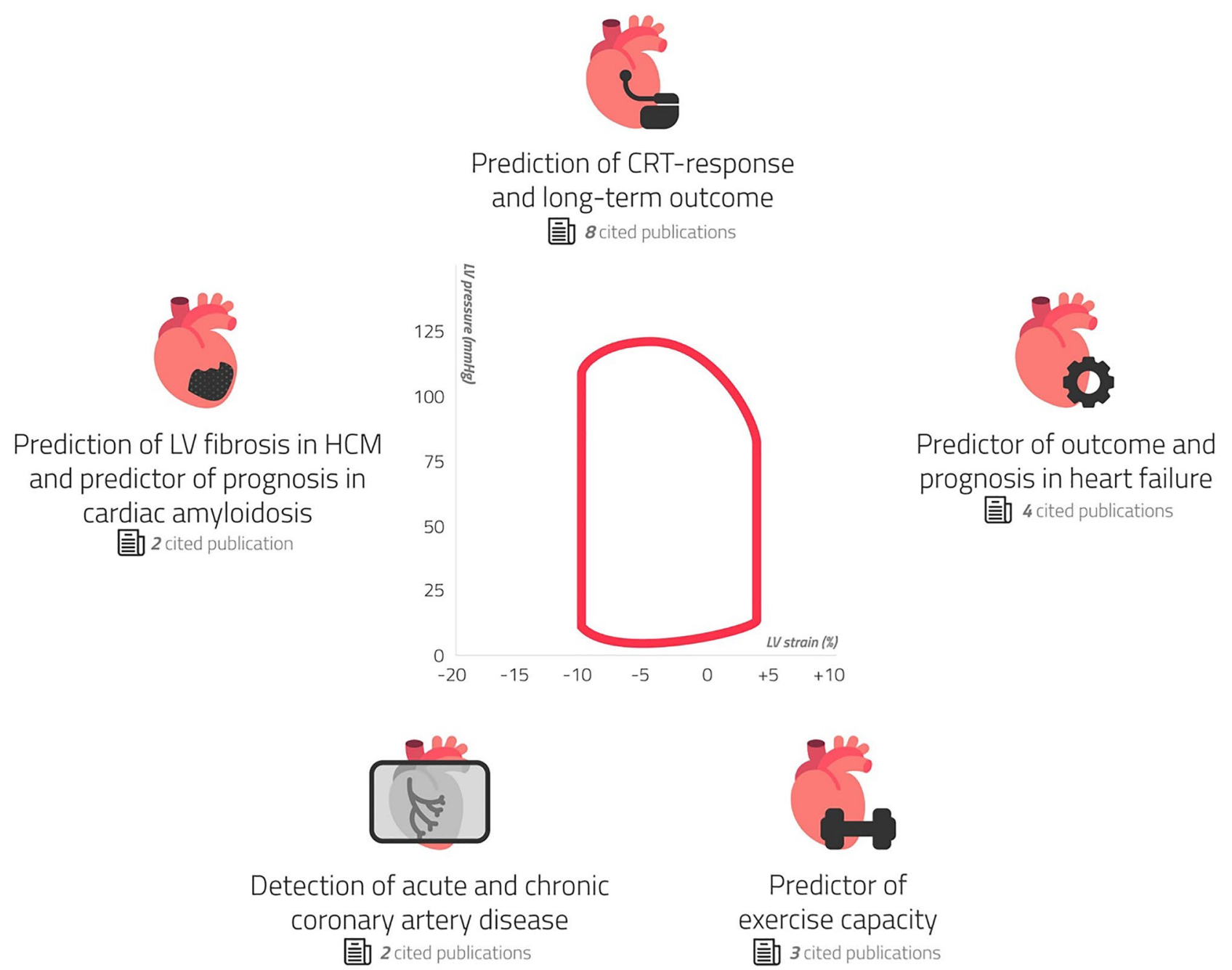

Fig. 5 Current scientific articles on myocardial work divided according to different clinical subtopics

reduction in GWW associated with LV reverse remodeling. Based on the findings of Russel et al. indicating that pressure strain measurements reflect cardiac metabolism, Galli et al. hypothesized that higher GCW at baseline represents a higher contractile reserve and is therefore able to predict positive CRT response, whereas decreased amount of GCW could be associated with reduced myocardial viability, limiting the beneficial effects of CRT [22, 36, 49, 50]. Furthermore, Ciampi et al. previously showed that the existence of contractile reserve assessed by dobutamine stress echocardiography is associated with a better prognosis, which is independent of the presence of LV dyssynchrony, as LV stimulation recruits viable myocardium [50]. There are several methods for the assessment of myocardial viability available, e.g., stress echocardiography, cardiac magnetic resonance, and nuclear imaging. Non-invasive estimation of myocardial work by pressure-strain analysis could provide a simple, easily accessible and cost-saving additive method.
Additionally, Galli et al. confirmed a predictive value of GWW for positive CRT response, postulating that GWW might stand for a recruitable energy waste which could be significantly reduced in positive CRT responders [36]. The combination of GCW > $1057 \mathrm{~mm} \mathrm{Hg} \%$ and GWW > $364 \mathrm{~mm}$ $\mathrm{Hg} \%$ showed very good specificity with low sensitivity [36]. Hence, myocardial work indices can be used to identify CRT responders, but patients might also benefit from CRT if GCW and GWW are below the mentioned cut-off values [36]. However, given the existence of numerous independent mechanisms influencing CRT response, the study suggests that the combination of clinical, electrocardiographic, and echocardiographic data in particular might be more useful to detect CRT responders [50-55]. In an earlier study by Vecera et al., also septal wasted work, especially in combination with the wall motion score index (WMSI), strongly predicted CRT response [56]. These results were confirmed in a study by Zhu et al. using a 3D echocardiography and 3D 
speckle tracking imaging analysis to simultaneously obtain left ventricular global and segmental principal strain and volume. Here, the measurement of baseline septal myocardial WW helped to enable a better patient selection for CRT (the more septal wasted at baseline, the higher probability of response to CRT was achieved) [57]. Also, there are preliminary data indicating that preserved GWE and GCW before CRT are associated with improved long-term outcome $[37,58]$. Recently, Duchenne et al. could demonstrate that the acute redistribution of regional myocardial work after CRT device implantation from the lateral to the septal wall appeared to be strongly related to long-term reverse remodeling. Proper patient selection should consider the presence of loading inhomogeneities for a successful resynchronization therapy [59] (Table 2). In a study by Kostyukevich et al., CRT responders had significantly larger septal WW and lateral CW compared to non-responders at baseline. Plus, on multivariate analysis, baseline lateral $\mathrm{CW}$ was independently associated with CRT response (CW $>881 \mathrm{~mm} \mathrm{Hg \% )}$ [60]. Moreover, CRT responders demonstrated a significant improvement in septal CW and WW as well as a decrease and an increase in lateral CW and WW respectively [60].

In conclusion, even though pressure strain loops and related indexes cannot be used on their own to assess CRT candidates, they represent a novel method that shows promising results in the prediction of CRT response and longterm outcomes.

\section{Ischemic heart disease}

In patients with non-ST-elevation myocardial infarction (NSTEMI), Boe et al. could show that the non-invasively estimated myocardial work index (MWI) was able to detect acute coronary occlusion, being superior to all other echocardiographic parameters used, including strain analysis [33]. Patients with NSTEMI represent a very heterogonous group in which timing of invasive therapy is not well defined [61, 62]. The identification of acute coronary occlusion in patients with NSTEMI could be beneficial in identifying patients who would benefit from direct or early revascularization [33].

In a further study, Edwards et al. validated that non-invasively assessed global myocardial work at rest allowed to detect subclinical coronary artery disease (CAD) in patients with preserved LVEF and no regional wall motion abnormalities (RWMAs) [34]. Interestingly, global myocardial work was even more sensitive in detecting subclinical coronary disease than global longitudinal strain (GLS), also in patients only suffering from a single-vessel disease [34]. GLS has a predominant contribution from the longitudinal arranged endocardial layer and therefore detects early ischemia-induced cardiac dysfunction since the subendocardium is more sensitive for reduced perfusion [63-70]. Other echocardiographic parameters that represent radial thickening as LVEF or RWMAs are less sensitive for the early derangement of myocardial function caused by ischemia [71, 72]. Here, global myocardial work performed even better than GLS underlining the sensitivity of myocardial work for myocardial oxygen consumption which seems to be reduced in early stages of CAD where LVEF is still preserved, and RWMAs are still absent [34].

In a retrospective analysis by El Mahdiui et al., a reduced myocardial work efficiency could be confirmed in patients with recently revascularized ST-elevation myocardial infarction and patients with heart failure with reduced ejection fraction [73]. However, there was no alteration of myocardial work efficiency in patients with no structural heart disease or patients presenting typical cardiovascular risk factors in comparison to healthy individuals [73] (Table 3).

\section{Chronic heart failure}

According to the current ESC Heart Failure Guidelines from 2016, heart failure represents a clinical diagnosis, which is characterized by typical symptoms and signs as well as increases of natriuretic peptide (BNP or NT-proBNP) [40].

Furthermore, echocardiographic determination of left ventricular function is necessary for the diagnosis of heart failure. Currently, left ventricular ejection fraction (LVEF) and also the assessment of left ventricular filling pressure by using the ratio of early transmitral flow and myocardial relaxation (E/e' ratio) are the recommended parameters of choice [40].

The prognostic accuracy of LVEF, while significant in the circumstance of an LVEF $<40 \%$, appears to be low in the case of HFpEF [74]. Additionally, LVEF is known to be significantly load dependent.

Today, myocardial strain measurements are well implemented in the daily clinical routine offering more precise, reproducible, and comprehensive information regarding $\mathrm{LV}$ mechanics and function. Clinical implications of myocardial strain assessment are diverse.

Notably, global longitudinal strain (GLS) was shown to be associated with outcome in symptomatic heart failure patients with reduced and preserved LVEF, and furthermore a stronger predictor of outcome than LVEF, especially in patients with preserved LVEF [75-78].

However, like LVEF, strain parameters prove to be dependent on afterload, resulting in a possible misinterpretation of the true contractile function [33, 79].

The estimation of myocardial work fixes this weakness by implementing the estimated LV pressure as described earlier.

In the setting of HFrEF, Wang et al. could show that global myocardial work (GMW) was a better prognosticator than both GLS and LVEF, where reduced values of GMW are significantly associated with death or poor outcome [80]. 


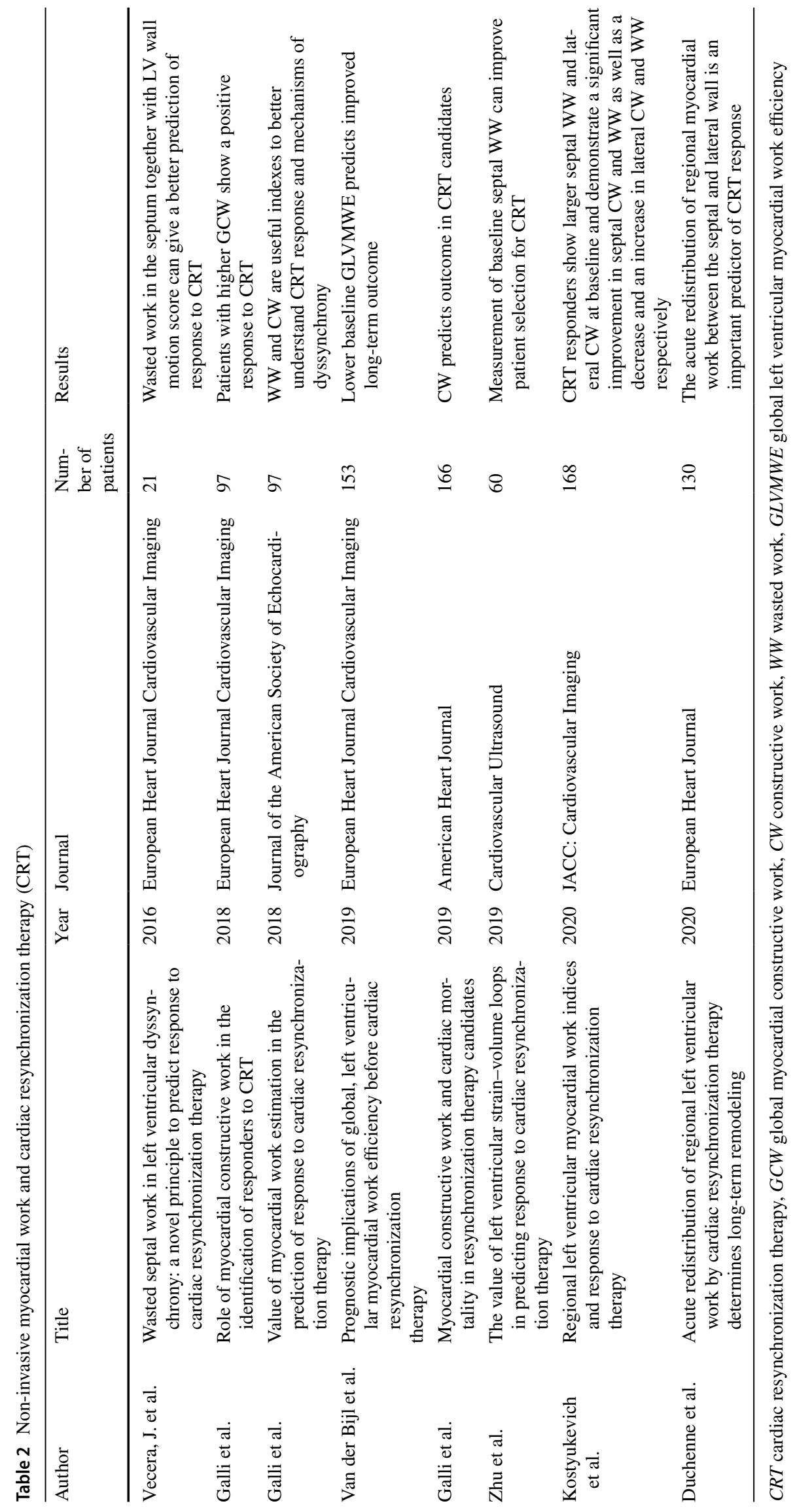




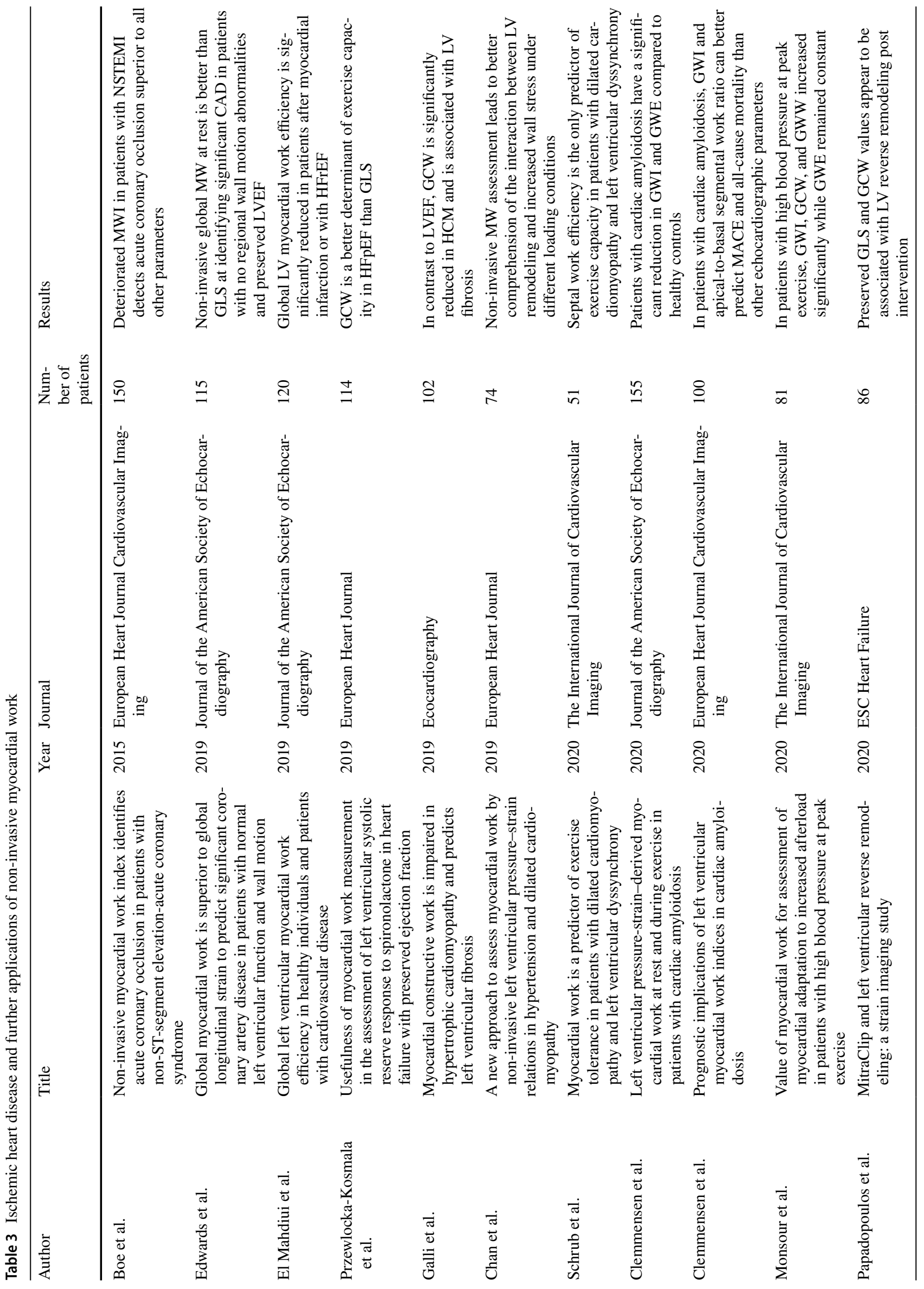




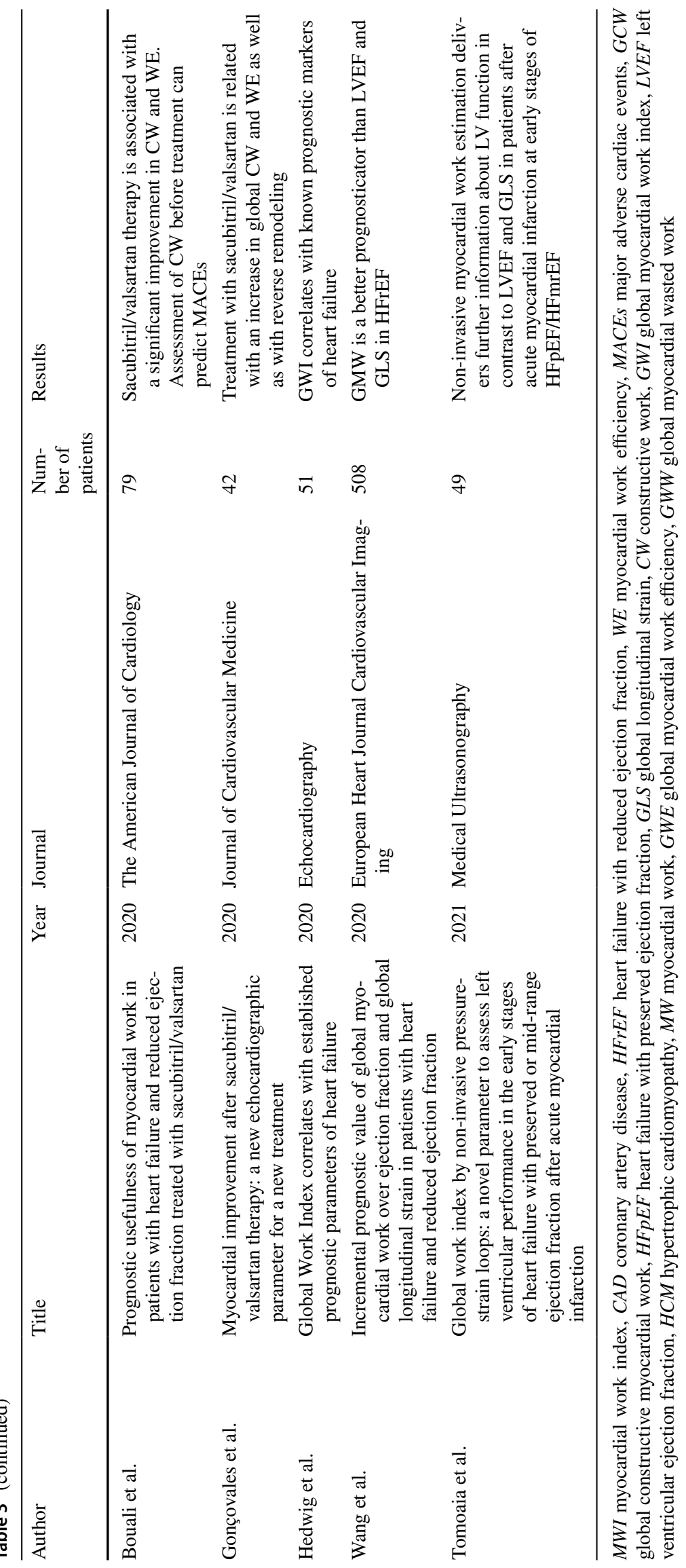


In a group of patients with acute myocardial infarction and heart failure with preserved or mid-range ejection fraction, it was shown that both GLS and Global Myocardial Work Index (GWI) are reduced in the majority of individuals. Some patients presented normal GWI despite abnormal GLS, emphasizing the importance of implementing blood pressure in the assessment of myocardial function [81].

Moreover, global constructive work (GCW) has been proven to be a better estimate of LV contractile response to physical effort, and hence a better measure of exercise capacity, in HFpEF than GLS. Its exertional increase in patients treated for 6 months with spironolactone is considered to be associated with improvement in functional capacity [82].

Heart failure patients with reduced ejection fraction treated with sacubitril/valsartan showed signs of LV reverse remodeling by common echocardiographic parameters as well as a significant improvement of constructive work and myocardial work efficiency during a follow up of 12 months. Wasted work, on the contrary, did not appear to be greatly affected [83, 84]. Also, GCW could predict long-term outcome in patients with HFrEF receiving sacubitril/valsartan. Not only was a GCW $\leq 910 \mathrm{mmHg}$ at baseline associated with a more advanced disease state, higher values for $\mathrm{LV}$ end-diastolic and end-systolic volume and more reduced LVEF but also a significant predictor of major adverse cardiac events (MACEs) before start of therapy [83]. Figure 6 demonstrates how changes in pressure-strain curves according to the LVEF range can be easily derived through the non-invasive assessment of MW by STE.

Hedwig et al. described the relation between global work index (GWI) and known prognostic parameters of echocardiography (GLS and LVEF), cardiopulmonary exercise test (Peak $\mathrm{O}_{2}$ consumption and $\mathrm{VE} / \mathrm{VCO}_{2}$ slope), and NTpro-BNP in patients with heart failure [85]. In particular, a GWI $<500 \mathrm{mmHg} \%$ could predict significant left ventricular remodeling, impaired LVEF, low exercise capacity, and high NT-pro-BNP levels, indicating dismal prognosis [85] (Table 3).

\section{Further applications of non-invasive myocardial work}

Recently, Chan et al. described various patterns of noninvasively assessed myocardial work by pressure-strain analysis in healthy patients and in those suffering from arterial hypertension or dilated cardiomyopathies [86]. Especially

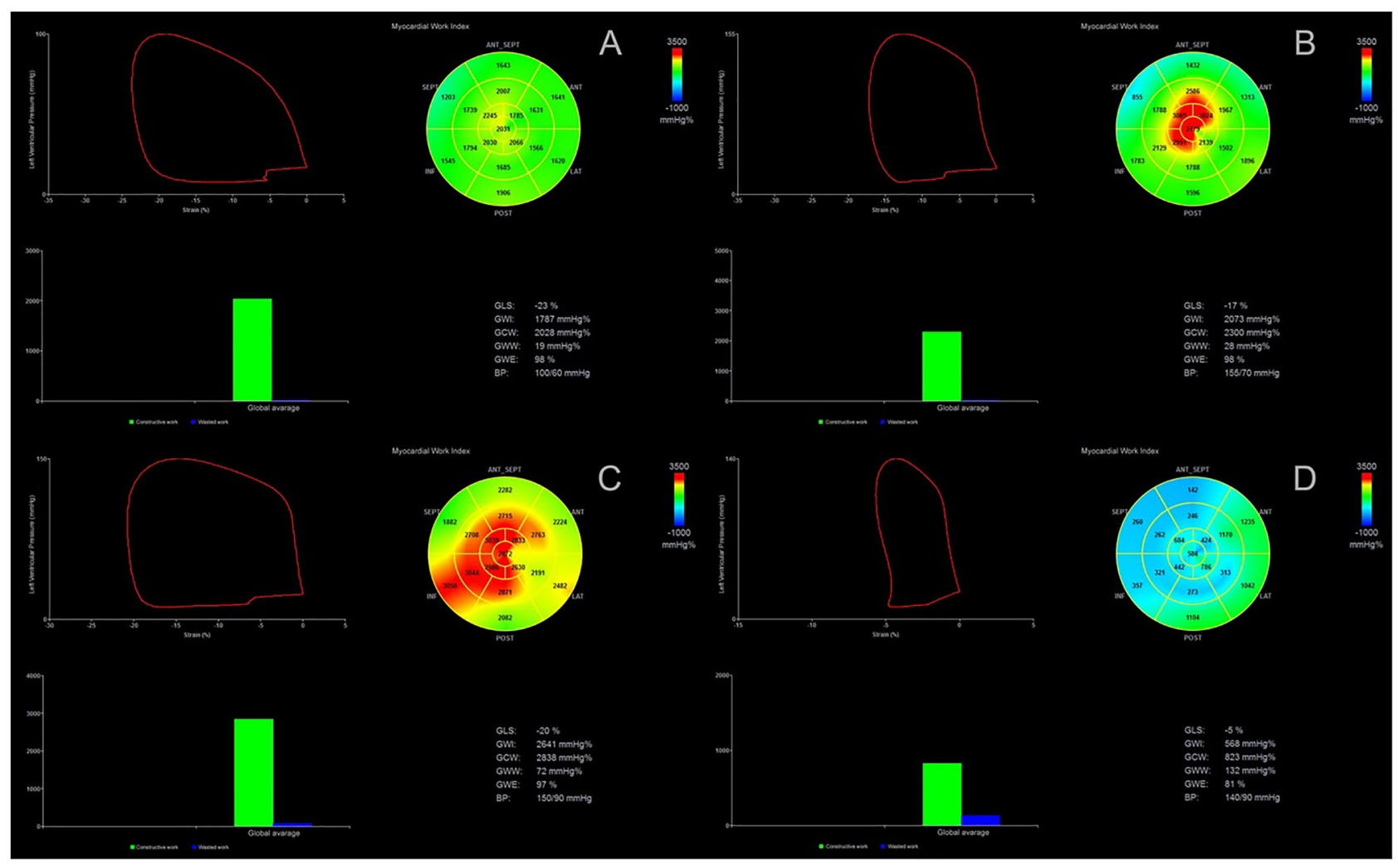

Fig. 6 Measurement of myocardial work indices by 2D echocardiography; representative pressure strain-loops, bull's eye plots of myocardial work index, and bar graphs showing GCW and GWW in control subjects (A) and subjects with $\operatorname{HFmrEF}(\mathbf{B}), \operatorname{HFpEF}(\mathbf{C})$, and HFrEF (D). GCW global constructive work, GWW global wasted work, HFmrEF heart failure with mid-range ejection fraction, HFpEF heart failure with preserved ejection fraction, HFrEF heart failure with reduced ejection fraction 
patients suffering from arterial hypertension presented exciting results: In comparison to the control group, high systolic blood pressure ( $>160 \mathrm{mmHg}$ ) led to a significant increase of GWI with no changes in MW efficiency, whereas GLS showed normal results [86]. These results confirm that usual STE-parameters as GLS are not able to reflect the increased cardiac energy demand to counteract increased afterload. Besides, in the cohorts of ischemic and not-ischemic dilated cardiomyopathy, there was a significant reduction of GWI and GWE due to an increase in wasted myocardial work and a reduction of constructive myocardial work [86].

Schrub et al. could also show in patients with dilated cardiomyopathy that the presence of left ventricular dyssynchrony assessed by echocardiography as septal flash or apical rocking leads to a significant reduction to global myocardial work efficiency due to an increase of wasted work [87]. These findings were especially present in the myocardial septum. Furthermore, the septal work efficiency was the only predictor of exercise capacity $\left(\mathrm{VO}_{2}\right.$ peak) in these patients [87].

Galli et al. were the first to investigate pressure-strain analysis in patients with non-obstructive hypertrophic cardiomyopathy [35]. Here, global constructive work appeared to be significantly impaired in comparison to healthy individuals, despite no significant changes in LVEF [35]. At a multivariable regression analysis, GCW emerged as the main predictor of $\mathrm{LV}$ fibrosis assessed by late gadolinium enhancement [35]. Furthermore, GCW correlated with $\mathrm{VO}_{2}$ peak assessed by cardiopulmonal exercise testing, favoring GCW as an indirect, easily accessible measure of exercise capacity in patients with non-obstructive HCM [35].

Mansour et al. studied the variations of MW indices during stress echocardiography in patients at peak exercise with high systolic blood pressure values of $>180 \mathrm{mmHg}$ [88]. They could confirm the results by Chan et al. [86], showing an increase of GCW, GWW, and GWI independent of peak GLS values at peak exercise, whereas GWE remained relatively preserved [88]. Furthermore, Mansour et al. investigated the contribution of the apex to MW. Here, the work done by the apex increased with peak exercise, emphasizing how the work done by the apex counteracts the energy loss measured by the GWW, especially in patients with $\mathrm{SBP}>180 \mathrm{mmHg}$ [88].

Non-invasive myocardial work has also been investigated in patients with cardiac amyloidosis at rest and during exercise by Clemmensen et al. [89]. These patients had significantly reduced measures of GWI and GWE at rest in comparison to healthy controls, especially pronounced in the basal segments of the myocardium. Furthermore, the myocardial work index reserve is significantly reduced in patients with cardiac amyloidosis in comparison to healthy individuals and correlates moderately with exercise capacity [89]. Another study by Clemmensen et al. could show that in patients with cardiac amyloidosis, GWI and the apicalto-segmental work ratio were able to predict MACE and all-cause mortality in these patients [90]. The new myocardial work indices outperformed GLS in predicting outcome, whereas GLS is already known to be more reliable than traditional echocardiographic parameters in the case of cardiac amyloidosis [90] (Table 3).

\section{Discussion}

\section{Reference values of myocardial work and correlation with standard/advanced 2DE parameters}

The NORRE (Normal Reference Ranges for Echocardiography) study, the first related multicenter study, investigated a large population of healthy individuals $(n=226)$ over a wide range of ages and delivered current normal reference values of non-invasive myocardial work indices [91]. Only in univariable analysis, GWI and GCW were age dependent. Merely in woman, both GWI and GCW increased with age due to an age-dependent significant increase of both systolic and diastolic blood pressure only in the female subgroup [91]. Moreover, multivariable analysis showed a gender and age independent correlation of both GWI and GCW only with systolic blood pressure [91]. Also, solely in univariable analysis, GWW and GWE showed independently of age minor as well as increased values in female individuals, respectively [91]. In conclusion, MW indices were not strongly dependent on age or gender. However, GWI and GWC were clearly associated with systolic blood pressure.

In a following sub study of the NORRE study, Manganaro et al. investigated the correlations between the new indices of non-invasive MW with main measures of systolic and diastolic function [92]. In multivariable analysis, the correlation between MW indices and LV size was not sufficiently significant. In fact, GWW and GWE poorly correlated with end systolic volume, while GWI and GCW were poorly correlated to LV mass adjusted to body surface area (BSA). These findings were not confirmed in multivariable analysis so that their relevance should be regarded as limited [92]. These results probably rely on the actual study population that only included healthy subjects, where LV size and consequentially parameters of cardiac function like MW indices are in normal ranges.

Similar considerations can be inferred when analyzing diastolic function: GWI and GCW correlated with LA size and $\mathrm{E} / \mathrm{E}^{\prime}$ ratio only in univariable analysis, leading to the consideration that $\mathrm{MW}$ and diastolic parameters are weakly associated [92]. Here, the Tei index made an exception, which proved to be significantly correlated with both GWW and GWE [92]. Moreover, both GWI and GCW were as expected associated with GLS, LVEF, and SBP as well 
as with global radial strain. In addition, GCW was also significantly correlated with global circumferential strain [92].

\section{Intra- and interobserver variability}

Several studies have tested the reproducibility of noninvasive myocardial work indices, showing good results regarding both intra- and inter-observer variability $[22,34,36,38$, $56,85,91]$

\section{Limitations of non-invasive pressure estimation}

Naturally, the LV pressure estimation cannot fully reproduce direct invasive measurements. Hence, pressure strain analysis comes with certain limitations which are essential for the understanding of this new method. Myocardial work by pressure strain analysis has to be understood as an index of cardiac work, as work is defined as the product of force and time. Pressure-strain analysis delivers a surrogate of the myocardial work performed by each segment, as LV pressure does not entirely explain each segment's force development [22]. When comparing left ventricles with different dimensions as in the study by Chan et al., myocardial work could be underestimated in dilated hearts due to their higher wall stress for each given LV-pressure [93, 94]. It is shown that the estimation of LV pressure itself is imprecise, leading to a bias of overestimation of the regional pressure-strain area [13]. Interestingly, Hubert et al. could show that the estimated global myocardial work parameters correlate very well with the invasively measured cardiac work [13].

In conditions of specific valvular pathologies, for example, aortic stenosis or left ventricular output obstruction, the estimation of LV peak pressure by measuring peripheral systolic arterial pressure cannot be used. The pressure gradient across the ventricle and the peripheral artery would lead to a false estimation of the actual LV pressure [22]. Regarding further disadvantages in comparison to invasive measurements, pressure-strain loop analysis is not able to deliver information about $\mathrm{LV}$ diastolic pressure or maximal rate of rise or fall of LV pressure [22].

Furthermore, since single blood pressure measurements can be very variable in the same patient during the day, this variability could eventually affect the assessment of myocardial work. The impact of taking a 24-h blood pressure measurement into account for the calculation of myocardial work needs further investigation. Also, strain analysis by echocardiography inherently depends on image quality which might have an impact on the feasibility of this method for some patients. In patients with cardiac arrhythmias, e.g., atrial fibrillation, GLS by STE might not be accurately assessable, questioning the estimation of myocardial work indices in these patients. Additionally, the estimation of myocardial work is software dependent and might differ among different vendors as it does for GLS [95]. Currently, the only software package available is provided by GE (Echopac V.202, GE). Boe et al. suggested that future improvements to the method, potentially by using 3D echocardiography, should include information about LV geometry, wall thickness, and local radii allowing the measurement of wall-stress instead of pressure [94]. Regarding the echocardiographic reference ranges, further studies in larger cohorts and different cardiac pathologies are needed. If the current results are valid for non-Caucasian European individuals is still uncertain [91].

\section{Prognostic value of non-invasive myocardial work assessment: contemporary and upcoming challenges}

As abovementioned, non-invasive assessment of myocardial work has been investigated in different cardiac conditions, ranging from coronary artery disease, including patients with NSTEMI, to LV-dyssynchrony, arterial hypertension, cardiac amyloidosis, and dilated and hypertrophic cardiomyopathy [33-36, 38, 56, 57, 82, 86-89]. However, to date, there are only a few small-scale studies which investigated the prognostic relevance of non-invasively assessed myocardial work, namely, studies on CRT and heart failure [37, $58,83,85]$. GCW and GWW, respectively as measures of cardiac reserve and energy loss, provide additional information to commonly used echocardiographic parameters in identifying CRT responders. The prospective, observational Contractile Reserve in Dyssynchrony (CRID) study investigated if wasted work can predict response to CRT in 210 participants who fulfill the indications of a CRTdevice implantation. The study was completed in December 2018, and the results are yet to be published [96]. In another ongoing study by Jens-Uwe Voigt et al. entitled "Myocardial Work and Metabolism in CRT (WORK-CRT)," non-invasive measures of LV mechanical dyssynchrony (e.g., apical rocking) will be examined to determine their predictive value in CRT response [97].

A further randomized interventional trial, "Early rhBNP on Myocardial Work in Patients With STEMI," by Song Ding et al. started in November 2019 and is set to end in September 2022, with an estimated number of 200 enrolled patients [98]. This study aims to assess the impact of early intracoronary injection and ongoing intravenous infusion of recombinant human BNP (rhBNP) over $72 \mathrm{~h}$ on myocardial work in patients with anterior STEMI after percutaneous coronary intervent [98]. The impact of non-invasively estimated myocardial work for the prediction of mortality in patients with acute myocardial infarction has yet to be determined. So far, pressure-strain analysis has not been investigated in patients with ST-segment elevation myocardial infarction. 
In patients with cardiogenic shock, measures of myocardial work were shown to be the strongest predictors of intra-hospital mortality, especially the cardiac power output (CPO) [99]. CPO is a parameter of external cardiac work, calculated as the product of cardiac output and mean aortic pressure divided by 451 . In a recently published study of our group, we could show that CPO precisely reflects left ventricular stroke work per minute over a broad scope of inotropic states [100]. Here, CPO was measured by right heart catheterization (RHC) as well as invasive arterial pressure which were correlated with LV SW $\mathrm{min}^{-1}$ measured via the conductance method, the invasive gold-standard. LVEF did not reflect myocardial work at all [100]. However, the use of RHC is controversial in critically ill patients [101]. The non-invasive estimation of myocardial work indices could be very useful to monitor cardiac function and inotropic interventions in the ICU setting.

Another interesting outlook is related to the field of transcatheter valve repair. Papadopoulos et al. conducted a retrospective case-control study to evaluate left ventricular global longitudinal strain and myocardial work indices in patients with heart failure and mitral regurgitation 1 year after transcatheter mitral-valve repair compared to patients treated with optimal medical treatment [102]. Preserved baseline GLS and GCW appeared to be able to predict reduction in LVEDV and LVESV, respectively, after successful MV repair proving to be potential new markers of positive LV remodeling, which could allow better patients' selection for this procedure [102]. In addition, patients who were treated with a MitraClip® showed stable GWE as well as improved GCW and GWI at follow-up, reflecting better cardiac energetics in contrast to patients treated with optical medical treatment [102].

\section{Conclusions}

The ability to assess myocardial work non-invasively broadens the currently available techniques for evaluating cardiac function. The adjustment to loading conditions leads to the implementation of critical hemodynamic concepts in the daily assessment of cardiac function. Further studies focusing on the prognostic impact of non-invasive myocardial work are needed.

Acknowledgements We would like to thank Simone Proietti Timperi for performing the graphical illustration.

Author contribution DA, TR AF, BP, CT, CZ, and AA contributed to the study conception and design. DA, TR, AF, and AA performed the literature research. DA, TR, AF, and DAM prepared the figures; DA, TR, AF, SK, CZ, and AA drafted the manuscript; all the authors edited and revised the manuscript and approved its final version.
Funding Open Access funding enabled and organized by Projekt DEAL.

\section{Declarations}

Competing interests DA reports no conflict of interest. TR reports no conflict of interest. AF is a shareholder of the Company BOCAhealthcare $\mathrm{GmbH}$. BP reports having received consultancy and lecture honoraria from Bayer, Daiichi Sankyo, MSD, Novartis, Sanofi-Aventis, Stealth Peptides, and Vifor Pharma; and editor honoraria from the Journal of the American College of Cardiology. BP and SK received funding from the DZHK (German Centre for Cardiovascular Research) and by the BMBF (German Ministry of Education and Research). Sebastian Kelle received an unrestricted research grant from Philips Healthcare and received lecture honoraria from Medis, NL. DAM reports no conflict of interest. CT has received speaker fees and/or contributions to congresses from Abbott, Abiomed, Astra Zeneca, Bayer, Berlin Chemie, Novartis, Pfizer, and Servier; all outside the submitted work. CZ reports no conflict of interest. AA reports no conflict of interest.

Open Access This article is licensed under a Creative Commons Attribution 4.0 International License, which permits use, sharing, adaptation, distribution and reproduction in any medium or format, as long as you give appropriate credit to the original author(s) and the source, provide a link to the Creative Commons licence, and indicate if changes were made. The images or other third party material in this article are included in the article's Creative Commons licence, unless indicated otherwise in a credit line to the material. If material is not included in the article's Creative Commons licence and your intended use is not permitted by statutory regulation or exceeds the permitted use, you will need to obtain permission directly from the copyright holder. To view a copy of this licence, visit http://creativecommons.org/licenses/by/4.0/.

\section{References}

1. Zaret BL, Wackers FJT, Terrin ML et al (1995) Value of radionuclide rest and exercise left ventricular ejection fraction in assessing survival of patients after thrombolytic therapy for acute myocardial infarction: results of thrombolysis in myocardial infarction (TIMI) phase II study. J Am Coll Cardiol 26:73-79. https://doi.org/10.1016/0735-1097(95)00146-Q

2. Burns RJ, Gibbons RJ, Yi Q et al (2002) The relationships of left ventricular ejection fraction, end-systolic volume index and infarct size to six-month mortality after hospital discharge following myocardial infarction treated by thrombolysis. J Am Coll Cardiol 39:30-36. https://doi.org/10.1016/S0735-1097(01) 01711-9

3. Nicolosi GL, Latini R, Marino P et al (1996) The prognostic value of predischarge quantitative two-dimensional echocardiographic measurements and the effects of early lisinopril treatment on left ventricular structure and function after acute myocardial infarction in the GISSI-3 trial. Eur Heart J 17:1646-1656. https:// doi.org/10.1093/oxfordjournals.eurheartj.a014747

4. Christian TF, Behrenbeck T, Gersh BJ, Gibbons RJ (1991) Relation of left ventricular volume and function over one year after acute myocardial infarction to infarct size determined by technetium-99m sestamibi. Am J Cardiol 68:21-26. https://doi. org/10.1016/0002-9149(91)90703-N

5. Galli E, Lancellotti P, Sengupta PP, Donal E (2014) LV mechanics in mitral and aortic valve diseases :value of functional 
assessment beyond ejection fraction. JACC Cardiovasc Imaging 7:1151-1166. https://doi.org/10.1016/j.jcmg.2014.07.015

6. Plana JC, Galderisi M, Barac A et al (2014) Expert consensus for multimodality imaging evaluation of adult patients during and after cancer therapy: a report from the American Society of Echocardiography and the European Association of Cardiovascular Imaging. Eur Heart J Cardiovasc Imaging 15:1063-1093. https://doi.org/10.1093/ehjci/jeu192

7. di Bella G, Minutoli F, Pingitore A et al (2011) Endocardial and epicardial deformations in cardiac amyloidosis and hypertrophic cardiomyopathy-2-D feature strain echocardiography. Circ J 75:1200-1208. https://doi.org/10.1253/circj.CJ-10-0844

8. Zito C, Manganaro R, Khandheria B et al (2015) Usefulness of left atrial reservoir size and left ventricular untwisting rate for predicting outcome in primary mitral regurgitation. Am J Cardiol 116:1237-1244. https://doi.org/10.1016/j.amjcard.2015.07.038

9. Donal E, Thebault C, Oconnor K et al (2011) Impact of aortic stenosis on longitudinal myocardial deformation during exercise. Eur J Echocardiogr 12:235-241. https://doi.org/10.1093/ ejechocard/jeq187

10. Kasner M, Aleksandrov A, Escher F et al (2017) Multimodality imaging approach in the diagnosis of chronic myocarditis with preserved left ventricular ejection fraction (MCpEF): The role of 2D speckle-tracking echocardiography. Int J Cardiol 243:374378. https://doi.org/10.1016/j.ijcard.2017.05.038

11. Tschöpe C, Senni M (2020) Usefulness and clinical relevance of left ventricular global longitudinal systolic strain in patients with heart failure with preserved ejection fraction. Heart Fail Rev 25:67-73. https://doi.org/10.1007/s10741-019-09853-7

12. Mor-Avi V, Patel MB, Maffessanti F et al (2018) Fusion of threedimensional echocardiographic regional myocardial strain with cardiac computed tomography for noninvasive evaluation of the hemodynamic impact of coronary stenosis in patients with chest pain. J Am Soc Echocardiogr 31:664-673. https://doi.org/10. 1016/j.echo.2018.01.019

13. Hubert A, Le Rolle V, Leclercq C et al (2018) Estimation of myocardial work from pressure-strain loops analysis: an experimental evaluation. Eur Hear J - Cardiovasc Imaging 19:1372-1379. https://doi.org/10.1093/ehjci/jey024

14. Burkhoff D, Mirsky I, Suga H (2005) Assessment of systolic and diastolic ventricular properties via pressure-volume analysis: a guide for clinical, translational, and basic researchers. Am J Physiol Heart Circ Physiol 289:H501-H512. https://doi.org/10. 1152/ajpheart.00138.2005

15. Kass DA, Yamazaki T, Burkhoff D et al (1986) Determination of left ventricular end-systolic pressure-volume relationships by the conductance (volume) catheter technique. Circulation 73:586-595. https://doi.org/10.1161/01.CIR.73.3.586

16. Kass DA, Midei M, Graves W et al (1988) Use of a conductance (volume) catheter and transient inferior vena caval occlusion for rapid determination of pressure-volume relationships in man. Cathet Cardiovasc Diagn 15:192-202. https://doi.org/10.1002/ ccd.1810150314

17. Westermann D, Kasner M, Steendijk P et al (2008) Role of left ventricular stiffness in heart failure with normal ejection fraction. Circulation 117:2051-2060. https://doi.org/10.1161/ CIRCULATIONAHA.107.716886

18. Suga H (1990) Ventricular energetics. Physiol Rev 70:247-277. https://doi.org/10.1152/physrev.1990.70.2.247

19. Suga $H$ (1979) Total mechanical energy of a ventricle model and cardiac oxygen consumption. Am J Physiol 236:H498-505. https://doi.org/10.1152/ajpheart.1979.236.3.H498

20. Baan J, van der Velde ET, de Bruin HG et al (1984) Continuous measurement of left ventricular volume in animals and humans by conductance catheter. Circulation 70:812-823
21. Kasner M, Sinning D, Burkhoff D, Tschöpe C (2015) Diastolic pressure-volume quotient (DPVQ) as a novel echocardiographic index for estimation of LV stiffness in HFpEF. Clin Res Cardiol 104:955-963. https://doi.org/10.1007/s00392-015-0863-y

22. Russell K, Eriksen M, Aaberge L et al (2012) A novel clinical method for quantification of regional left ventricular pressurestrain loop area: a non-invasive index of myocardial work. Eur Heart J 33:724-733. https://doi.org/10.1093/eurheartj/ehs016

23. Morris JJ, Pellom GL, Murphy CE et al (1987) Quantification of the contractile response to injury: assessment of the work-length relationship in the intact heart. Circulation 76:717-727. https:// doi.org/10.1161/01.cir.76.3.717

24. Safwat A, Leone BJ, Norris RM, Foëx P (1991) Pressure-length loop area: its components analyzed during graded myocardial ischemia. J Am Coll Cardiol 17:790-796. https://doi.org/10. 1016/S0735-1097(10)80199-8

25. Rushmer RF, Franklin DL, Ellis RM (1956) Left ventricular dimensions recorded by sonocardiometry. Circ Res 4:684-688. https://doi.org/10.1161/01.RES.4.6.684

26. Miyamoto MI, Kim CS, Guerrero JL et al (1999) Ventricular pressure and dimension measurements in mice. Lab Anim Sci 49:305-307

27. Pasipoularides A, Shu M, Shah A et al (2002) Right ventricular diastolic function in canine models of pressure overload, volume overload, and ischemia. Am J Physiol Circ Physiol 283:H2140 H2150. https://doi.org/10.1152/ajpheart.00462.2002

28. Leraand S, Kiil F (1969) Local dimensional changes of the myocardium measured by ultrasonic technique. Scand J Clin Lab Invest 24:361-371. https://doi.org/10.3109/00365516909080172

29. Delhaas T, Arts T, Prinzen FW, Reneman RS (1998) Estimates of regional work in the canine left ventricle. Prog Biophys Mol Biol 69:273-287. https://doi.org/10.1016/S0079-6107(98)00012-1

30. Skulstad H, Edvardsen T, Urheim S et al (2002) Postsystolic shortening in ischemic myocardium: active contraction or passive recoil? Circulation 106:718-724. https://doi.org/10.1161/01.CIR. 0000024102.55150.B6

31. Urheim S, Rabben SI, Skulstad H et al (2005) Regional myocardial work by strain Doppler echocardiography and LV pressure: a new method for quantifying myocardial function. Am J Physiol Hear Circ Physiol 288:2375-2380. https://doi.org/10.1152/ajpheart. 00946.2004

32. Russell K, Eriksen M, Aaberge L et al (2013) Assessment of wasted myocardial work: a novel method to quantify energy loss due to uncoordinated left ventricular contractions. Am J Physiol Circ Physiol 305:H996-H1003. https://doi.org/10.1152/ajpheart. 00191.2013

33. Boe E, Russell K, Eek C et al (2015) Non-invasive myocardial work index identifies acute coronary occlusion in patients with non-ST-segment elevation-acute coronary syndrome. Eur Hear J - Cardiovasc Imaging 16:1247-1255. https://doi.org/10.1093/ ehjci/jev078

34. Edwards NFA, Scalia GM, Shiino K et al (2019) Global myocardial work is superior to global longitudinal strain to predict significant coronary artery disease in patients with normal left ventricular function and wall motion. J Am Soc Echocardiogr 32:947-957. https://doi.org/10.1016/j.echo.2019.02.014

35. Galli E, Vitel E, Schnell F et al (2019) Myocardial constructive work is impaired in hypertrophic cardiomyopathy and predicts left ventricular fibrosis. Echocardiography 36:74-82. https://doi. org/10.1111/echo. 14210

36. Galli E, Leclercq C, Fournet M et al (2018) Value of myocardial work estimation in the prediction of response to cardiac resynchronization therapy. J Am Soc Echocardiogr 31:220-230. https://doi.org/10.1016/j.echo.2017.10.009

37. Galli E, Hubert A, Le Rolle V et al (2019) Myocardial constructive work and cardiac mortality in resynchronization therapy 
candidates. Am Heart J 212:53-63. https://doi.org/10.1016/j. ahj.2019.02.008

38. Galli E, Leclercq C, Hubert A et al (2018) Role ofmyocardial constructive work in the identification of responders to CRT. Eur Heart J Cardiovasc Imaging 19:1010-1018. https://doi.org/ 10.1093/ehjci/jex191

39. Wells G, Parkash R, Healey JS et al (2011) Cardiac resynchronization therapy: a meta-analysis of randomized controlled trials. CMAJ 183:421-429. https://doi.org/10.1503/cmaj.101685

40. Ponikowski P, Voors AA, Anker SD et al (2016) 2016 ESC guidelines for the diagnosis and treatment of acute and chronic heart failure. Eur Heart J 37:2129-2200. https://doi.org/10.1093/ eurheartj/ehw128

41. Chung ES, Leon AR, Tavazzi L et al (2008) Results of the Predictors of Response to CRT (PROSPECT) trial. Circulation 117:2608-2616. https://doi.org/10.1161/CIRCULATIONAHA. 107.743120

42. Ypenburg C, van Bommel RJ, Borleffs CJW et al (2009) Longterm prognosis after cardiac resynchronization therapy is related to the extent of left ventricular reverse remodeling at midterm follow-up. J Am Coll Cardiol 53:483-490. https://doi.org/10. 1016/j.jacc.2008.10.032

43. van Bommel RJ, Tanaka H, Delgado V et al (2010) Association of intraventricular mechanical dyssynchrony with response to cardiac resynchronization therapy in heart failure patients with a narrow QRS complex. Eur Heart J 31:3054-3062. https://doi. org/10.1093/eurheartj/ehq334

44. Yu C-M, Fung W-H, Lin H et al (2003) Predictors of left ventricular reverse remodeling after cardiac resynchronization therapy for heart failure secondary to idiopathic dilated or ischemic cardiomyopathy. Am J Cardiol 91:684-688. https://doi.org/10.1016/ s0002-9149(02)03404-5

45. Søgaard P, Egeblad H, Kim WY et al (2002) Tissue Doppler imaging predicts improved systolic performance and reversed left ventricular remodeling during long-term cardiac resynchronization therapy. J Am Coll Cardiol 40:723-730. https://doi.org/10. 1016/s0735-1097(02)02010-7

46. Arshad A, Moss AJ, Foster E et al (2011) Cardiac resynchronization therapy is more effective in women than in men: the MADIT-CRT (Multicenter Automatic Defibrillator Implantation Trial with Cardiac Resynchronization Therapy) trial. J Am Coll Cardiol 57:813-820. https://doi.org/10.1016/j.jacc.2010.06.061

47. McLeod CJ, Shen W-K, Rea RF et al (2011) Differential outcome of cardiac resynchronization therapy in ischemic cardiomyopathy and idiopathic dilated cardiomyopathy. Hear Rhythm 8:377-382. https://doi.org/10.1016/j.hrthm.2010.11.013

48. Park MY, Altman RK, Orencole M et al (2012) Characteristics of responders to cardiac resynchronization therapy: the impact of echocardiographic left ventricular volume. Clin Cardiol 35:779780. https://doi.org/10.1002/clc.22043

49. Reant P, Zaroui A, Donal E et al (2010) Identification and characterization of super-responders after cardiac resynchronization therapy. Am J Cardiol 105:1327-1335. https://doi.org/10.1016/j. amjcard.2009.12.058

50. Ciampi Q, Pratali L, Citro R et al (2009) Identification of responders to cardiac resynchronization therapy by contractile reserve during stress echocardiography. Eur J Heart Fail 11:489496. https://doi.org/10.1093/eurjhf/hfp039

51. Parsai C, Bijnens B, Sutherland GR et al (2009) Toward understanding response to cardiac resynchronization therapy: left ventricular dyssynchrony is only one of multiple mechanisms. Eur Heart J 30:940-949. https://doi.org/10.1093/eurheartj/ehn481

52. Brunet-Bernard A, Maréchaux S, Fauchier L et al (2014) Combined score using clinical, electrocardiographic, and echocardiographic parameters to predict left ventricular remodeling in patients having had cardiac resynchronization therapy six months earlier. Am J Cardiol 113:2045-2051. https://doi.org/10.1016/j. amjcard.2014.03.050

53. Lafitte S, Reant P, Zaroui A et al (2009) Validation of an echocardiographic multiparametric strategy to increase responders patients after cardiac resynchronization: a multicentre study. Eur Heart J 30:2880-2887. https://doi.org/10.1093/eurheartj/ehn582

54. Delgado V, van Bommel RJ, Bertini M et al (2011) Relative merits of left ventricular dyssynchrony, left ventricular lead position, and myocardial scar to predict long-term survival of ischemic heart failure patients undergoing cardiac resynchronization therapy. Circulation 123:70-78. https://doi.org/10.1161/ CIRCULATIONAHA.110.945345

55. Lim P, Buakhamsri A, Popovic ZB et al (2008) Longitudinal strain delay index by speckle tracking imaging: a new marker of response to cardiac resynchronization therapy. Circulation 118:1130-1137. https://doi.org/10.1161/CIRCULATIONAHA. 107.750190

56. Vecera J, Penicka M, Eriksen M et al (2016) Wasted septal work in left ventricular dyssynchrony: a novel principle to predict response to cardiac resynchronization therapy. Eur Hear J - Cardiovasc Imaging 17:624-632. https://doi.org/10.1093/ ehjci/jew019

57. Zhu M, Chen H, Fulati $Z$ et al (2019) The value of left ventricular strain-volume loops in predicting response to cardiac resynchronization therapy. Cardiovasc Ultrasound 17:3. https:// doi.org/10.1186/s12947-019-0153-3

58. van der Bijl P, Vo NM, Kostyukevich MV et al (2019) Prognostic implications of global, left ventricular myocardial work efficiency before cardiac resynchronization therapy. Eur Hear J - Cardiovasc Imaging 31:1388-1394. https://doi.org/10.1093/ ehjci/jez095

59. Duchenne J, Aalen JM, Cvijic M et al (2020) Acute redistribution of regional left ventricular work by cardiac resynchronization therapy determines long-term remodelling. Eur Hear J - Cardiovasc Imaging 32:619-628. https://doi.org/10.1093/ ehjci/jeaa003

60. Kostyukevich MV, van der Bijl P, Vo NM et al (2020) Regional left ventricular myocardial work indices and response to cardiac resynchronization therapy. JACC Cardiovasc Imaging. https:// doi.org/10.1016/j.jcmg.2020.03.006

61. Sorajja P, Gersh BJ, Cox DA et al (2010) Impact of delay to angioplasty in patients with acute coronary syndromes undergoing invasive management. analysis from the ACUITY (Acute Catheterization and Urgent Intervention Triage strategY) trial. J Am Coll Cardiol 55:1416-1424. https://doi.org/10.1016/j.jacc. 2009.11.063

62. Jneid H, Anderson JL, Wright RS et al (2012) 2012 ACCF/AHA focused update of the guideline for the management of patients with unstable angina/non-st-elevation myocardial infarction (updating the 2007 guideline and replacing the 2011 focused update). Circulation 126:875-910. https://doi.org/10.1161/CIR. 0b013e318256f1e0

63. Choi J-O, Cho SW, Bin SY et al (2009) Longitudinal 2D strain at rest predicts the presence of left main and three vessel coronary artery disease in patients without regional wall motion abnormality. Eur J Echocardiogr 10:695-701. https://doi.org/10.1093/ ejechocard/jep041

64. Montgomery DE, Puthumana JJ, Fox JM, Ogunyankin KO (2012) Global longitudinal strain aids the detection of non-obstructive coronary artery disease in the resting echocardiogram. Eur Heart J Cardiovasc Imaging 13:579-587. https://doi.org/10.1093/ ejechocard/jer282

65. Yingchoncharoen T, Agarwal S, Popović ZB, Marwick TH (2013) Normal ranges of left ventricular strain: a meta-analysis. J Am Soc Echocardiogr 26:185-191. https://doi.org/10.1016/j. echo.2012.10.008 
66. Tsai W-C, Liu Y-W, Huang Y-Y et al (2010) Diagnostic value of segmental longitudinal strain by automated function imaging in coronary artery disease without left ventricular dysfunction. J Am Soc Echocardiogr 23:1183-1189. https://doi.org/10.1016/j. echo.2010.08.011

67. Dahlslett T, Karlsen S, Grenne B et al (2014) Early assessment of strain echocardiography can accurately exclude significant coronary artery stenosis in suspected non-ST-segment elevation acute coronary syndrome. J Am Soc Echocardiogr 27:512-519. https://doi.org/10.1016/j.echo.2014.01.019

68. Sjøli B, Ørn S, Grenne B et al (2009) Diagnostic capability and reproducibility of strain by Doppler and by speckle tracking in patients with acute myocardial infarction. JACC Cardiovasc Imaging 2:24-33. https://doi.org/10.1016/j.jcmg.2008.10.007

69. Shimoni S, Gendelman G, Ayzenberg O et al (2011) Differential effects of coronary artery stenosis on myocardial function: the value of myocardial strain analysis for the detection of coronary artery disease. J Am Soc Echocardiogr 24:748-757. https://doi. org/10.1016/j.echo.2011.03.007

70. Winter R, Jussila R, Nowak J, Brodin L-A (2007) Speckle tracking echocardiography is a sensitive tool for the detection of myocardial ischemia: a pilot study from the catheterization laboratory during percutaneous coronary intervention. J Am Soc Echocardiogr 20:974-981. https://doi.org/10.1016/j.echo. 2007.01.029

71. Hanekom L, Cho G-Y, Leano R et al (2007) Comparison of twodimensional speckle and tissue Doppler strain measurement during dobutamine stress echocardiography: an angiographic correlation. Eur Heart J 28:1765-1772. https://doi.org/10.1093/ eurheartj/ehm 188

72. Reimer KA, Jennings RB (1979) The "wavefront phenomenon" of myocardial ischemic cell death. II. Transmural progression of necrosis within the framework of ischemic bed size (myocardium at risk) and collateral flow. Lab Invest 40:633-644

73. El Mahdiui M, van der Bijl P, Abou R et al (2019) Global left ventricular myocardial work efficiency in healthy individuals and patients with cardiovascular disease. J Am Soc Echocardiogr 32:1120-1127. https://doi.org/10.1016/j.echo.2019.05.002

74. Solomon SD, Anavekar N, Skali H et al (2005) Influence of ejection fraction on cardiovascular outcomes in a broad spectrum of heart failure patients. Circulation 112:3738-3744. https://doi.org/ 10.1161/CIRCULATIONAHA.105.561423

75. Huang W, Chai SC, Lee SGS et al (2017) Prognostic factors after index hospitalization for heart failure with preserved ejection fraction. Am J Cardiol 119:2017-2020. https://doi.org/10.1016/j. amjcard.2017.03.032

76. Sengel $\varnothing v$ M, Jørgensen PG, Jensen JS et al (2015) Global longitudinal strain is a superior predictor of all-cause mortality in heart failure with reduced ejection fraction. JACC Cardiovasc Imaging 8:1351-1359. https://doi.org/10.1016/j.jcmg.2015.07. 013

77. Kalam K, Otahal P, Marwick TH (2014) Prognostic implications of global LV dysfunction: a systematic review and metaanalysis of global longitudinal strain and ejection fraction. Heart 100:1673-1680. https://doi.org/10.1136/heartjnl-2014-305538

78. Stanton T, Leano R, Marwick TH (2009) Prediction of all-cause mortality from global longitudinal speckle strain. Circ Cardiovasc Imaging 2:356-364. https://doi.org/10.1161/CIRCIMAGING.109.862334

79. Donal E, Bergerot $\mathrm{C}$, Thibault $\mathrm{H}$ et al (2009) Influence of afterload on left ventricular radial and longitudinal systolic functions: a two-dimensional strain imaging study. Eur J Echocardiogr J Work Gr Echocardiogr Eur Soc Cardiol 10:914-921. https://doi. org/10.1093/ejechocard/jep095

80. Wang CL, Chan YH, Wu VCC et al (2020) Incremental prognostic value of global myocardial work over ejection fraction and global longitudinal strain in patients with heart failure and reduced ejection fraction. Eur Hear J - Cardiovasc Imaging 348356. https://doi.org/10.1093/ehjci/jeaa162

81. Tomoaia R, Beyer RS, Zdrenghea D et al (2020) Global work index by non-invasive pressure-strain loops: a novel parameter to assess left ventricular performance in the early stages of heart failure with preserved or mid-range ejection fraction after acute myocardial infarction. Med Ultrason 23:62-69. https://doi.org/ 10.11152/mu-2672

82. Przewlocka-Kosmala M, Marwick TH, Mysiak A et al (2019) Usefulness of myocardial work measurement in the assessment of left ventricular systolic reserve response to spironolactone in heart failure with preserved ejection fraction. Eur Heart J Cardiovasc Imaging 20:1138-1146. https://doi.org/10.1093/ehjci/ jez027

83. Bouali Y, Donal E, Gallard A et al (2020) Prognostic usefulness of myocardial work in patients with heart failure and reduced ejection fraction treated by sacubitril/valsartan. Am J Cardiol. https://doi.org/10.1016/j.amjcard.2020.03.031

84. Valentim Gonçalves A, Galrinho A, Pereira-da-Silva $\mathrm{T}$ et al (2020) Myocardial work improvement after sacubitril-valsartan therapy: a new echocardiographic parameter for a new treatment. J Cardiovasc Med (Hagerstown) 21:223-230. https://doi.org/10. 2459/JCM.0000000000000932

85. Hedwig F, Soltani S, Stein J et al (2020) Global work index correlates with established prognostic parameters of heart failure. Echocardiography 37:412-420. https://doi.org/10.1111/echo. 14612

86. Chan J, Edwards NFA, Khandheria BK et al (2019) A new approach to assess myocardial work by non-invasive left ventricular pressure-strain relations in hypertension and dilated cardiomyopathy. Eur Heart J Cardiovasc Imaging 20:31-39. https:// doi.org/10.1093/ehjci/jey131

87. Schrub F, Schnell F, Donal E, Galli E (2019) Myocardial work is a predictor of exercise tolerance in patients with dilated cardiomyopathy and left ventricular dyssynchrony. Int J Cardiovasc Imaging. https://doi.org/10.1007/s10554-019-01689-4

88. Mansour MJ, AlJaroudi W, Mansour L et al (2020) Value of myocardial work for assessment of myocardial adaptation to increased afterload in patients with high blood pressure at peak exercise. Int J Cardiovasc Imaging. https://doi.org/10.1007/ s10554-020-01867-9

89. Clemmensen TS, Eiskjær H, Mikkelsen F et al (2020) Left ventricular pressure-strain-derived myocardial work at rest and during exercise in patients with cardiac amyloidosis. J Am Soc Echocardiogr 33:573-582. https://doi.org/10.1016/j.echo.2019. 11.018

90. Clemmensen TS, Eiskjær H, Ladefoged B et al (2020) Prognostic implications of left ventricular myocardial work indices in cardiac amyloidosis. Eur Hear $\mathbf{J}$ - Cardiovasc Imaging 1-10. https://doi.org/10.1093/ehjci/jeaa097

91. Manganaro R, Marchetta S, Dulgheru R et al (2019) Echocardiographic reference ranges for normal non-invasive myocardial work indices: results from the EACVI NORRE study. Eur Heart J Cardiovasc Imaging 20:582-590. https://doi.org/10.1093/ehjci/ jey 188

92. Manganaro R, Marchetta S, Dulgheru R et al (2019) Correlation between non-invasive myocardial work indices and main parameters of systolic and diastolic function: results from the EACVI NORRE study. Eur Hear J - Cardiovasc Imaging 32:1-9. https:// doi.org/10.1093/ehjci/jez203

93. Chan J, Edwards NFA, Khandheria BK et al (2019) A new approach to assess myocardial work by non-invasive left ventricular pressure-strain relations in hypertension and dilated cardiomyopathy. Eur Hear J - Cardiovasc Imaging 20:31-39. https:// doi.org/10.1093/ehjci/jey131 
94. Boe E, Skulstad H, Smiseth OA (2019) Myocardial work by echocardiography: a novel method ready for clinical testing. Eur Heart J Cardiovasc Imaging 20:18-20. https://doi.org/10.1093/ ehjci/jey156

95. Farsalinos KE, Daraban AM, Ünlü S et al (2015) Head-to-head comparison of global longitudinal strain measurements among nine different vendors: the EACVI/ASE inter-vendor comparison study. J Am Soc Echocardiogr 28:1171-1181.e2. https://doi.org/ 10.1016/j.echo.2015.06.011

96. US National Library of Medicine (2015) Contractile reserve in dyssynchrony: a novel principle to identify candidates for cardiac resynchronization therapy (CRID-CRT). In: ClinicalTrials.gov. https://clinicaltrials.gov/ct2/show/NCT02525185

97. US National Library of Medicine (2015) Myocardial work and metabolism in CRT (WORK-CRT). In: ClinicalTrials.gov. https://clinicaltrials.gov/ct2/show/NCT02537782

98. US National Library of Medicine (2019) The Impact of Early rhBNP on myocardial work in patients with anterior ST-segment elevation myocardial infarction after percutaneous coronary intervention. In: Clinical Trials.gov. https://clinicaltrials.gov/ ct2/show/NCT04157868
99. Fincke R, Hochman JS, Lowe AM et al (2004) Cardiac power is the strongest hemodynamic correlate of mortality in cardiogenic shock: a report from the SHOCK trial registry. J Am Coll Cardiol 44:340-348. https://doi.org/10.1016/j.jacc.2004.03.060

100. Abawi D, Faragli A, Schwarzl M et al (2019) Cardiac power output accurately reflects external cardiac work over a wide range of inotropic states in pigs. BMC Cardiovasc Disord 19:217. https:// doi.org/10.1186/s12872-019-1212-2

101. Shah MR, Hasselblad V, Stevenson LW et al (2005) Impact of the pulmonary artery catheter in critically ill patients. JAMA 294:1664. https://doi.org/10.1001/jama.294.13.1664

102. Papadopoulos K, Ikonomidis I, Chrissoheris M et al (2020) MitraClip and left ventricular reverse remodelling: a strain imaging study. ESC Hear Fail. https://doi.org/10.1002/ehf2.12750

Publisher's Note Springer Nature remains neutral with regard to jurisdictional claims in published maps and institutional affiliations. 